\title{
The eyeglass reversal
}

\section{Songjoo Oh}

Published online: 30 March 2011

(C) Psychonomic Society, Inc. 2011
Sometimes, if not frequently, people notice that an object looks ambiguous or reversible in some perceptual features of it, such as depth or meaning. For example, when aFerris wheel in an amusement park is seen from a long distance, it is unclear whether its left side is nearer or farther away than the right side. The silhouette of a person walking on a river bank at sunset is ambiguousas to whether he/she is stepping forward or backward. Or, in a soccer game seen on television, a ball kicked into the air can be seen as flying either nearer or farther away. This phenomenon has been given various names in perception research, such as ambiguity, reversibility, or multistability, and many types of examples have been found or devised.

It has been widely believed that these examples involve fundamentalaspects of visual perception,such as perceptual organization (Attneave, 1971; Leopold \& Logothetis, 1999; Long \& Toppino, 2004; Pomerantz \& Kubovy, 1986). Accordingly, many investigators have extensively studied a variety of factors that influence perceptual reversal, and the factors are often categorized into either bottom-up classes or top-down classes (see Long \& Toppino, 2004, for a review). Canthese factors account for reversals that the naïve population voluntarily experiences in daily life? So far, previous studies have been limited in explaining the initial reversal of a real object, and there are at least two reasons for this.

First, in the majority of previous laboratoryexperiments, those factors have been tested under the assumption that all humanshavean ability for skilled reversal. Prior to each experiment, participants were askedwhether theyknew how experimental visual stimuli could be ambiguous and what the alternative forms were. However, this assumption seems to have limitations for the generalization of those results to the voluntary reversal that a naïve observer may experience in daily life, since people are not usually skilled at seeing reversals. Indeed, Rock and his colleagues found thatunless 
they were informed of the reversibilityof an intrinsically reversible object, most people failed to see the reversal itself, so that they usually persisted with the initial organizations of reversible figures (Girgus, Rock, \& Egatz 1977; Rock, Hall, \& Davis, 1994; Rock \& Mitchener, 1992). Accordingly, they suggestedthat different processes might be involved in subsequent/repeated reversals and initial reversals.

Some studies have shown that initial reversals appear to be more related to effortful cognitive processes, such as problem solving, inference, decision making, or working memory maintenance (Reisberg, 1983; Rock, 1975, 1983; Rock et al., 1994), while subsequent reversals seem to use automatic sensory processes, such as neural fatigue or adaptation (Köhler, 1947; Pastukhov \& Braun, 2007; Pearson \& Brascamp, 2008). As a result, they differ in the measurement of observers' responses. Laboratory experimentsfor initial reversals usually measure whether or not observers succeed in seeing a reversal. In contrast, the experiment of subsequent reversal measureshow many times in a given duration observers alternate their percepts or how long they maintain a specific alternative. Because of these differences between initial and subsequent reversals, it is difficult to suggest that the results from the studies of subsequent reversals can be directly applied to account for everyday reversals.

Second, there have been some studies regarding initial reversals, most of which were done by Rock and his colleagues (Girgus et al., 1977; Rock et al., 1994; Rock \& Mitchener, 1992). However, they used only drawing versions of ambiguous figures to test their ideas. One limitation of their stimuli is that they may have been too far from reality. For example, in those figures, some natural perceptual features, such as color, shape, and surface, were simplified as lines or were even removed. Furthermore, to make the figures look more ambiguous, their shapes were distorted andmade unrealistic. Their studies may have had some limitations in explaining the reversal of a real object. Therefore, one wonders if their findings can be directly adapted to account for reversals of everyday objects, which have concrete perceptual features.

Of course, there have been a number of studies testing perceptual reversals by using real objects, such as the Necker cube (e.g. Hochberg \& Peterson, 1987; Long \& Toppino, 2004; Peterson \& Hochberg, 1983). However, in all those studies, only subsequent reversals were tested. Namely, observers'intention was manipulated with specific knowledge on how a reversible object was ambiguous, and the observers had to be able to carry it out prior to the experiments.

Considering the limitations that these two types of studies have for the reversal of a real object, a remaining research question is the following: How do people achieve an initial reversal voluntarily from a real object? Examining this question more thoroughly can be expected to provide some ideas that account for those reversals that people voluntarily notice with objects in the real world.

\section{Experiment}

For an initial reversal, naïve observers' intentionshave been tested and have been expected to play a crucial role in achieving a reversal (Girgus et al., 1977; Rock et al., 1994; Rock \& Mitchener, 1992). Theseresearchers differentiated the specificity of intentions by giving differing instructions that provided either general knowledge or specific knowledge about reversals (Rock et al., 1994). In other words, the intention of an observer to see a reversible figure could be formed either with general information about it,such asthe figure can be seen in an ambiguous way, or with concrete information,such ashow it is ambiguous and what the alternatives are. Rock et al. presented naïve observers with a Necker cubelike figure reversible in perspective and found that observers with intention and general knowledge about it succeeded in seeing it slightly more often than did observers who passively observed the figure with no information (63\% vs. $50 \%$; see their preliminary Experiment 1). In contrast, when the same observers were given specific knowledge, almostall of them succeeded in seeing the reversal (94\%). Thus, they suggested that specific knowledge may play a critical role in an initial reversal of an ambiguous figure.

In the present study, it was tested how intention would influence the initial reversal of a real object. For this purpose, a new reversal was manipulated by placing a pair of eyeglasses in the air. As is shown in Fig. 1a, the reversal can easily be demonstrated with a regular pair of eyeglasses. If the eyeglasses are seen at arm distance with one eye, the eyeglasses can be reversible in perspective in two ways: The lenses may appear farther away than the legs, as they actually are (veridical percept), or the lenses may look nearer than the legs, as if the eyeglasses are resting on a book (illusory percept), as shown in Fig. 1 b.

\section{Method}

Participants A total of 250 collegestudents participated in this experiment. All had normal or corrected-to-normal vision andwere naïveas to the purpose of the study. Also, all the participants had not experienced the eyeglassreversal before.

Apparatus and stimui $\mathrm{A}$ pair of regular metal eyeglasses $(13 \mathrm{~cm}$ wide $\times 7.83 \mathrm{~cm}$ high $\times 15 \mathrm{~cm}$ long)was presented on the top of a bamboo stick placed on a table. The eyeglasses were viewed inwardly or outwardly, as shown in 
Fig. 1 A simple demonstration of eyeglass reversal. a The legs are located closer than the lenses. b The same eyeglasses can be seen in an illusory way, so that the lenses look nearer than the legs, which form is analogous to a pair of eyeglasses resting on a book
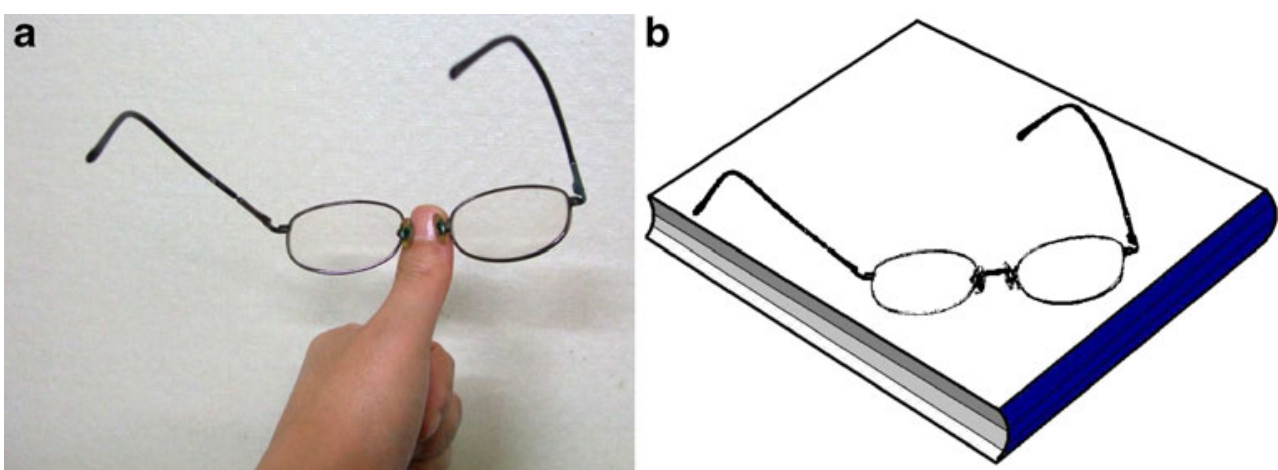

Fig. 2. The outwardly viewed direction of the eyeglasses wasproduced simply by turning the inwardly viewed eyeglasses about $180^{\circ}$. The eyeglasses were placed so as not to have any occlusion cues between the legs and the lenses. The observer saw the eyeglasses through a rectangular-shaped peephole $(1.5 \times 1.0 \mathrm{~cm})$ with one eye closed, so that binocular depth cues were not available. The viewing distance between the peephole and the lens of the eyeglasses was set at $96 \mathrm{~cm}$. Observers were asked to hold their headsas stationary as possible during the observation. Before the observation, two ambiguous figureswere shown to participants for the purpose of demonstratingthe concept of perceptual reversals and differentiating their knowledge. The two figures includedblack-and-white line drawing versions of the Necker cube and the rabbit-duck ambiguous figure. Each of the figures was printed on a regular sheet of white paper, seen on a desk in front of the observer. It had been hypothesized that the Necker cube induces the eyeglass reversal more efficiently than does the rabbitduck figure, since the Necker cube reversal is more analogous to the eyeglass reversal than is the rabbit-duck reversal in the content of reversibility, as had been demonstrated with reversible drawings (Rock et al., 1994).
Design Two hundred participants were randomly assigned to one of eight separate groups, consisting of the types of viewing direction (inward or outward), instructions (intention or nointention), and prior figures (the Necker cube or the rabbit-duck ambiguous figure). Thus, each condition had 25 separate students, and the design was a $2 \times 2 \times 2$ independent-group factorial design, with a one-trial observation method.In the intention condition, participants weretold that the eyeglasses were reversible in some way and were asked to try to find it. But they were not informed of how,specifically, the eyeglasses were reversible. The instructionsto the participants were as follows: "Please focus your eyes on the eyeglasses, and reportanything, as quickly as possible, that may be seen as reversible.You may refer to the reversal of the Necker cube (or the rabbit-duck figure) you saw previously." In contrast, participants in the no-intention condition were instructed that they were going to complete a memory test in which they hadto draw the Necker cube or the rabbit-duck ambiguous figure immediatelyafter observing the eyeglasses. In addition to these four experimental conditions, anothernew 50 observers participated in two control conditions: 25 were assigned to the inwardly and 25 to the outwardly viewed conditions. For

Fig. 2 a Illustration of the inward direction of the eyeglasses where the legs are closer to the peephole than the lenses. b Frontal view of the inwardly viewed condition. c Frontal view of the outwardly viewed condition
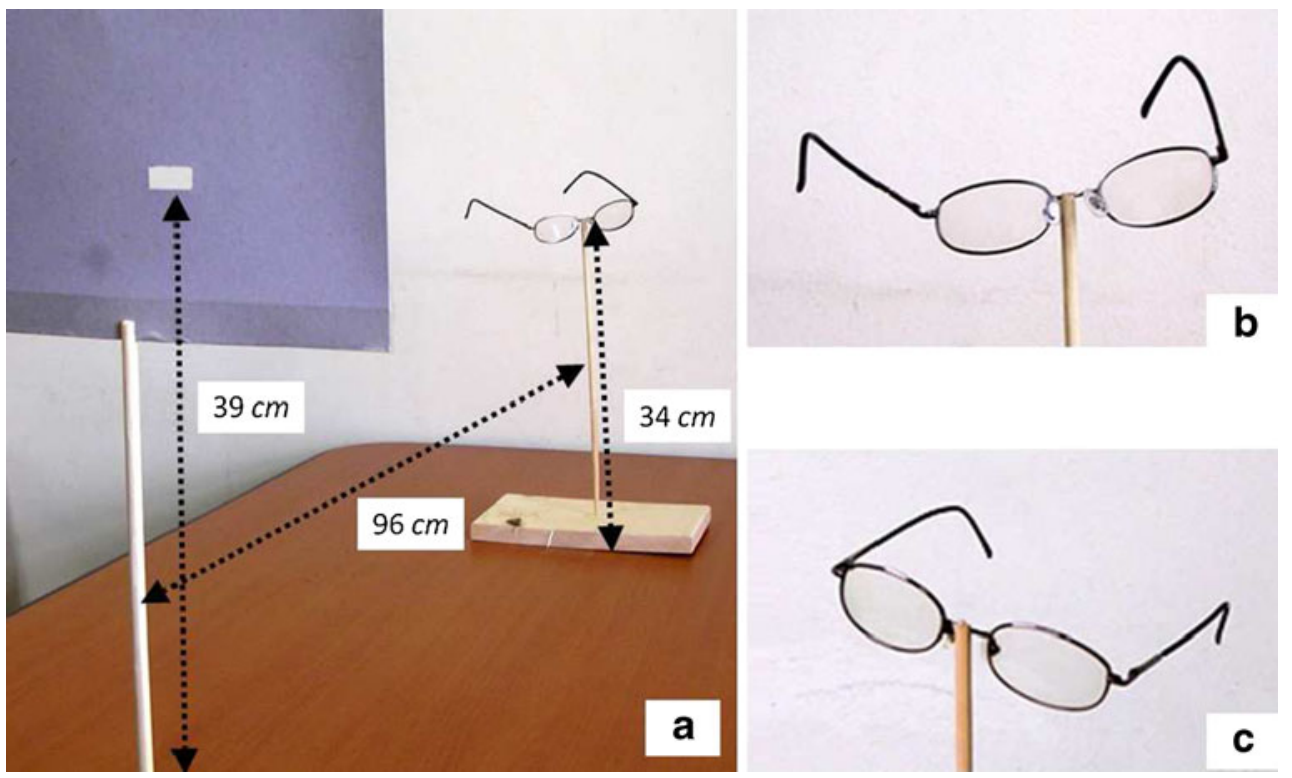
the control group, no line drawing prime figures were shown, and the observers were instructed to look carefully at the eyeglasses for $60 \mathrm{~s}$.Also, they were told that the experimenter would ask them a question about the eyeglasses afterward.

Lastly, the observers who had not achieved the eyeglass reversal during the main observation session in all the conditions aboveparticipated in an additional observation session. In this posthoc observation session, they were presented with the same eyeglasses in the same observation condition again for the $60 \mathrm{~s}$ duration, but this time they were informed that the eyeglasses were actually reversible in perspective and were asked to try to see it in that way.For example, they were informed that the legs of the eyeglasses could be seen as farther than the lenses in the outwardly viewed condition. Thus, their intention to see the reversal was formed with a specific knowledge about it.

Procedure Before observing the eyeglasses, each participant in the experimental conditions were shown either the Necker cube or a rabbit-duck ambiguous figure printed on paper, and the experimenter explained verbally how they were ambiguous. The observers were allowed to see the figures until they were able to voluntarily switch between the alternatives. They confirmed that theyeach understood the concept of a perceptualreversal, and only those who had actually experienced the reversal participated in the eyeglass observation session. All of the participants in the rabbit-duck prime conditions were familiar with the figure prior to the task, which facilitated their understanding of the reversal concept. Onlyone fourth of the participants in the Necker cube prime conditions did not see the reversal or the two alternative perspectives immediately. Accordingly, two more unambiguous cubes, having occlusion cues, were presented to the participants until they learned to see its reversal, which took from a few seconds to minutes, depending on theindividual. Afterward, each participant was seated in front of the experiment table, and they were allowed to see the eyeglasses for $60 \mathrm{~s}$. The experimenter measured the time taken with a stopwatch. If participants correctly reported the reversal, the observation time ended. Otherwise, the observationcontinued for the full $60 \mathrm{~s}$. The participantsin the no-intention conditions and the participants who failed to see the reversal were interviewed immediately after the observation session to ascertain whether or not they had seen the reversal. Usually, when participants saw the reversal, they also had an "aha"reaction and confidently reportedthat they saw the reversal. They were all interviewed and were askedwhich organization between the two alternative views of the eyeglasses they had had first in their mind at the beginning of the observation. After the interview, observers who had failed to see the eyeglass reversalparticipated in the posthoc observation sessionand were given specific knowledge about the session.

\section{Results}

Each observer reported whether or not he/she achieved the eyeglass reversal and then was categorized as either a"success" or a "failure." For statistical analysis, a success was coded 1 , while a failure was coded 0 . The results for all the conditions are shown in Fig. 3. The mostinteresting finding is an asymmetric pattern for the success rate of the eyeglass reversal between the inwardly and outwardly viewed directions. Only 7 out of 125 participants (5.6\%) achieved the eyeglass reversal through all outwardly viewed conditions regardless of intention, which is in sharp contrast to the 66 participants who successfully achieved the reversal $(52.8 \%)$ in the inwardly viewed condition. The

Fig. 3 Number of observers who achieved the eyeglass reversal in the main observation session. The values given in each bracket indicate the observers who were successful in the posthoc observation session. The total number of the observers who obtained the reversal in each condition should be the sum of the two figures

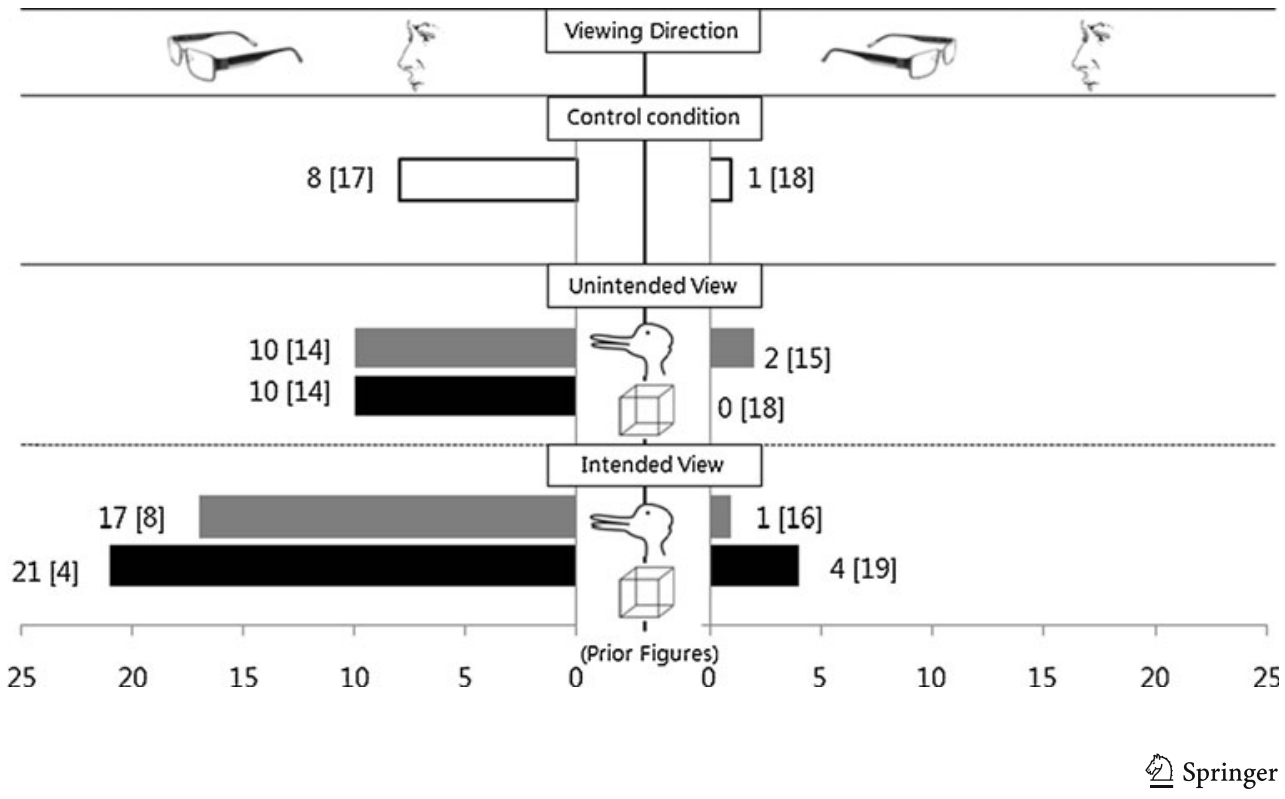


difference between the two views was significant, Pearson $\chi^{2}(1, N=100)=75.09, p=.000$. A log-linear analysis was conducted to evaluate the relational effect of three category variables (viewing orientation $\times$ intention $\times$ prior figure) on the success or failure frequency of the eyeglass reversal, excluding the control condition. Among these comparisons, only the two-way interaction between direction and intention was significant, Pearson $\chi^{2}(1)=4.546, p=.031$, indicating that the effect of intention differs depending on the viewing direction. This result could be attributed to the fact that very few observers achieved the eyeglass reversal in the outwardly viewed condition, and further analyses for it seem to be unnecessary. Thus, the statistical results are described below only for the inwardly viewed condition.

For the inwardly viewed condition (excluding the control condition), a two-way contingency table analysis was conducted to evaluate whether intention correlated with the pattern of successfor the eyeglass reversal. The result showed a significant difference between the intention and no-intention conditions, Pearson $\chi^{2}(1, N=100)=13.300$, $p=.000$, indicating that intention had a significant effecton the eyeglass reversal. No significant differences were shown between the no-intention and the control conditions, Pearson $\chi^{2}(2, N=75)=0.456, p=.796$. This result suggests that experiencing an ambiguous figure immediately before the experiment does not increase the rate of the success of the reversal.

For theintention conditionin the inwardly viewed condition, the number of observers who achieved the reversal did not significantly differ between the Necker cube and rabbit-duck prior figureconditions (21 vs. 17), Pearson ${ }^{2}(1, N=50)=.657$, $p=.418$. On the other hand, when the elapsed time of the eyeglass reversal was analyzed, the observers who experienced theNecker cube figure prior to the task were significantly fasterthan the participants who saw therabbitduck ambiguous figure (12.7 vs. $21.7 \mathrm{~s}$, on average), and a Mann-Whitney $U$ test showed a significant differencealso, $z=2.217, p=.027$ (in this analysis, only achievers' data were used). The Necker cube group had an average rank of 15.90, while the rabbit-duck group had an average rank of 23.94, indicating that the rabbit-duck figure was less effective for priming the reversal than was the Necker cube.

At first, 7 of the participants reportedthatthey had viewed the lenses as nearer than the legs but soon realized that this interpretation must be incorrect (because the nosepads of the eyeglasses were seen in the front of the lenses) andcorrected their interpretation. These participant reports support previous studies showing that observers' intention to reverse global forms could be influenced by localocclusion cues (Peterson \& Hochberg 1983). It was assumed, however, that the advantages of little occlusion cues might be similarly effective across all conditions, so that it might be controlled.
Regarding the initial organization of the eyeglasses, 89 out of $118(72 \%)$ observers in all the inwardly viewed conditions, including the control condition, and 124 out of $125(99.2 \%)$ observersin the outwardly viewed condition, who remembered their initial organizations of the 3-D structure of the eyeglasses, reported that, at the beginning of the observation, they had seenthe lenses as closer than the legs-namely, asthe outward direction.

In the posthoc observation sessions, in which observers who had not achieved the eyeglass reversal in the main observation session participated and in which these observers were informed of the eyeglass reversal with specific information about it, all except 2 observersachieved the eyeglass reversal in the inwardly viewed and outwardly viewed conditions, respectively. Thus, $98 \%$ of the observers in the inwardly viewed condition and $75 \%$ of the observers in the outwardly viewed condition achieved the eyeglass reversal. This difference was significant, Pearson $\chi^{2}(1, N=$ $200)=22.650, p=.000$. In boththecontrol conditions, similarly, 25 observers $(100 \%)$ in the inwardly viewed condition and 19 observers (76\%) in the outwardly viewed condition achieved the eyeglass reversal, Pearson $\chi^{2}(1, N=$ $50)=6.818, p=.009$. The significant difference between the two viewing conditions indicates that the outwardly viewed condition was more difficult for achieving the reversal.

\section{Discussion}

The aim of the present study was to examine how people achieve the very first reversal of a real object. For this purpose, a pair of regular eyeglasses, reversible in perspective, were presented to naïve observers in monocular vision under situationsin which the specificity of intention and viewing directions were varied. The results largely support previous findings showing the crucial role of intention in the reversal. However, it seems that the specificity of intention that is required for the reversal differs according to the viewing condition of the eyeglasses.

First, the Necker cube,which was analogous to the eyeglasses in the content of perceptualambiguity, induced much faster reversal than did the rabbit-duck ambiguous figure. This result is commensurate with previous findings showing that past experience or expectation of a form influences the perceptual organization of a following similar type of form (Balcetis \& Dunning, 2010; Boring, 1930; Rock et al., 1994; Sterzer, Frith, \& Petrovic, 2008). Thus, experiencing a reversible figure drawn on paper can be transferred into the reversal of threedimensional real objects. Second, the results for the outwardly viewed condition suggest that intention works only when it is accompanied with specific knowledge about the reversal. 
On the other hand, the results, especially from the inwardly viewed condition, are a little controversial. First, intention withgeneral knowledge on the reversibility of eyeglasses successfully induced the reversal. In previous studies, Rock and his colleagues suggested that intention with general knowledge was insufficient for the achievement of an initial reversal (Girgus et al., 1977; Rock et al., 1994; Rock \& Mitchener, 1992). Second, it should be pointed out that a considerable number of observers in the no-intention condition and the controlcondition for the inwardly viewed eyeglasses achieved the reversalat a rate that was much higher than that for the outwardly viewed intention condition. This finding indicates the importance of the stimulus variable in the experience of an initial reversal.

Considering these points together, an internal factor (intention) and an external factor (viewing direction) seem to cooperatively influence an initial reversal of eyeglasses. It has been known that these two factors are important for the case of subsequent or skilled reversals also (e.g., Hochberg \& Peterson, 1987). Butthis does not necessarily mean that a common mechanism is involved in both types of reversibility; rather,it seems that stimulus factors were disregarded in the previous studies of initial reversals. This suggests that intentionality might be overemphasized. Three factors other than intentionality that are thought to influence eyeglass reversal should also be considered in detail: depth cue availability, formal familiarity, and formal typicality.

Depth cue availability Above all, the question is what made the two viewing conditions for the eyeglasses differ in the effect of intentionality. Especially, why was it so difficult for observers in the outwardly viewed condition to see the eyeglass reversal with general information about it? In a way, this result can be interpreted as a floor effect. More specifically, the outwardly viewed eyeglasses might not be ambiguous at all, so that the effect of observers' intention was not sensitively revealed. One potential cause for it is the depth information of the eyeglasses. For example, some pictorial depth cues, such as occlusion, blur, linear perspective, and kinetic depth cues caused by observers' slight head movement, might have been highly possible in this condition, as compared with the inwardly viewed condition, so that the lenses looked closer with respect to thelegs than they actually were. Accordingly, it might be difficult for observers to overcome those cues and find the eyeglass reversal, with only intention formed by general knowledge about it. Instead, a more elaborated intention, such as that formed by specific information about the reversal, might be needed for overcoming those cues to achieve it. As Gibson (1966) famously argued, indeed, such ambiguity do not occur in the real world, in which depth cues are evident. Thus, the very few reversals in the outwardly viewed condition might be nothing more than a natural example of an exact perception. According to this explanation, depth information for the inwardlyviewed eyeglasses might have been deficient, so that observers experienced more reversals, even without intention. Certainly, this view seems to account for such reversals experienced in the real world when depth cues are less clear due to foggy weather, far distance, and littleocclusion cue.

But, although the depth cue explanation may be true in a fully natural situation, there is room for doubt about applying it to the results of the present study. Considering that these depth cues are generally immediately effective (Gibson, 1979) and the observational conditions of the eyeglasses, such as viewing distance and orientation, were carefully controlled almost the same for the inwardly viewed condition, it is dubious that only depth cues made the two viewing conditions that much different. In addition, in the posthoc observation session, a totalof $75 \%$ of the observers succeeded in seeing the reversal with both general and specific information about it in the outwardly viewed condition. This also raises doubt that the difficulty in seeing the eyeglass reversal in the outwardly viewed condition resulted simply from any depth cues, since it is generally hard to intentionally alternate the depth relationship of objects.

Formal familiarity Another potential factor might be deeply related to the fact that observers' initial organization of the eyeglasses was biased for the outwardly viewed direction across the two viewing conditions. Why did so many observers see the eyeglasses placed toward them in the two viewed directions? This might be because they were accustomed to seeing eyeglasses in the direction in which eyeglasses are worn on others' faces - the outwardly viewed direction. Indeed, it has been extensively demonstrated that familiarity plays a crucial role in the perceptual organization of familiar objects, such as the body and face. For example, the back view ofa point-light walker is more likely to look like the front view (Vanrie, Dekeyser, \& Verfaillie, 2004), body joints ambiguous in direction appear more normal in direction (Oh, 2009), and a hollow face looks like a normal convex face (Gregory, 1990).

Considering these findings, the outwardly viewed bias of the eyeglasses may not be surprising. Some possible reasons could be the depth cues mentioned above or other cues; in the inwardly viewed condition, the initial, incorrect, organization might have been "caught" and corrected to fit with the veridical organization, while, in the outwardly viewed condition, the observers were stuck in the initial, correct, organization, without reversal. Meanwhile, observers' intention or some uncontrolled depth cues might have additionally increased both possibilities in each condition. In traditional studies on the reversal of drawn versions of ambiguous figures, whether 
initial organization is correct or not is meaningless because the concept of correctness is indefinable. However, it can be problematic in the real world. Nevertheless, it seems to be obvious that how people organize eyeglassesthe very first time is crucial for inducing the reversal of such a real object.

Formal typicality Lastly, both inwardly and outwardly viewed eyeglasses might differ from each other in the equivalency of reversibility of their forms. More specifically, the inwardly viewed eyeglasses might have been less typical as a view, such that this direction might have been more ambiguous or unstable. In contrast, the outwardly viewed eyeglasses might have been more typical as a view, such that it was far less susceptible to the effect of the intention about the reversal. Accordingly, the observers were more likely to hold that organization stably.

In most previous studies, however, formal or configural typicality has been treated seriously. Especially, in the drawing versions of reversible figures, ambiguities were subjectively manipulated or distorted by the experimenter so as to be as evenly ambiguous across alternatives as possible. For example, in order for the Necker cubeto look more ambiguous in depth, the oblique lines depicted as receding in depth are often drawn parallel, without linear perspective. Thus, in the perception of such ambiguous stimuli, the role of observers' cognitive factors, such as knowledge, intention, and expectation, might become relatively important. The pure role of such cognitive factors in perceptual organization might have been concealing much more sensitivity by usingambiguous figures. However, this kind of cube may look odd in the real world. On the other hand, the eyeglasses tested in the present study were presented normally, without any artificial transformation. From this point of view, the eyeglassesmay have been seen in a more culturally possible form, and their configural typicality in perspective was much clearer,especially in the outwardly viewed condition.

Altogether, three possible factors have been considered that might have contributed to the results of the present study that differ from those of previous studies showing the effect of intention,especiallythose done by Rock and his colleagues. But the factors remain as hypotheses for further studies. In the present study, the observational conditions for the eyeglasses stimulus were seminatural rather than perfectly natural. Participantsobserved the eyeglasses in specific directions, with their heads stationary, using one eye and for prolonged observation duration. Consequently, more frequent reversals might have occurred than in the real world, in which depth information is redundant. Indeed, very few people would see the eyeglass reversal in ordinary life. It may be almost impossible for anyone to achieve the eyeglasses reversal in naturally viewed sit- uations, in which binocular vision and motion cues are available, despite a strong intention with specific knowledge about it. Thus, the difference in reversals between the inwardly viewed and outwardly viewed eyeglasses seems to indicate both extreme effects:the observers' factor and the stimulus factor.

Recently, popular models of perceptual reversal have been hybrid models that emphasize the interaction between low-level sensory processes and high-level cognitive processes (Leopold \& Logothetis, 1999; Lig et al., 2008; Long \& Toppino, 2004; Sterzer, Kleinschmidt, \& Ress, 2009). Within this framework, the results of the present study can be understood on a continuumincluding the two processes:The larger the effect of cognitive factors is, the weaker the sensory factors are, or vice versa. Yet most of those studies are based on subsequent reversals, in which observers already know all the alternatives of the reversible figures. Probably, when an observer sees a reversible figure, such as the Necker cube, hypothetically direct neural connections between the alternatives may not be formed until the observer experiences the reversal of the figure, as has been suggested elsewhere (Rock, 1975, 1983; Rock et al., 1994). But the more the observer experiences the reversal, the stronger the neural connections become. Accordingly, the neural activity for subsequent reversals may differ from that for initial reversals. From that point of view, rather, the eyeglass reversal in the present study may need to be understood asan insight-like process, in that it occurs abruptly and spontaneously. Unfortunately, it seems that no study has tried to understand initial perceptual reversals at a neurological level, although some studies have been done for cognitive insights, such as in riddles or verbal problems (Jung-Beeman et al., 2004; Luo \& Niki, 2003). This puzzling issue should be investigated more in further studies.

Acknowledgements This work was supported by Kyungnam University Research Fund, 2011. I also wish to thank Joshua Solomon and three anonymous reviewers for their comments on earlier versions of this article and a special thanks to John Franchak and Sapna Prasad for their proofreading.

\section{References}

Attneave, F. (1971). Multistability in perception. Scientific American, $225,63-71$.

Boring, E. G. (1930). A new ambiguous figure. American Journal of Psychology, 42, 444.

Balcetis, E., \& Dunning, D. (2010). Wishful seeing: motivational influences on visual perception of the physical environment. In E. Balcetis, \& D. G. Lassiter (Eds.), Social psychology of visual perception. New York: Psychology Press.

Braun, J., \& Pastukhov, A. (2007). Perceptual reversals need no prompting by attention. Journal of Vision, 7(10, Art. 5), 1-17. 
Gibson, J. J. (1966). The senses considered as perceptual systems. Boston: Houghton Mifflin.

Gibson, J. J. (1979). The ecological approach to visual perception. Boston: Houghton Mifflin.

Girgus, J. J., Rock, I., \& Egatz, R. (1977). The effect of knowledge of reversibility on the reversibility of ambiguous figures. Perception \& Psychophysics, 22, 550-556.

Gregory, R. L. (1990). Eye and brain: The psychology of seeing. New York: McGraw-Hill.

Hochberg, J., \& Peterson, M. A. (1987). Piecemeal organization and cognitive components in object perception: Perceptually coupled responses to moving objects. Journal of Experimental Psychology: General, 116, 370-380.

Jung-Beeman, M., Bowden, E. M., Haberman, J., Frymiare, J. L., Abambel-Liu, S., Greenblatt, R., ...Kounios, J. (2004). Neural activity when people solve verbal problems with insight. PLoS Biology, 2(4), 0500-0510.

Köhler, W. (1947). Gestalt psychology: An introduction to new concepts in modern psychology. New York: Liverright.

Leopold, D. A., \& Logothetis, N. K. (1999). Multistable phenomena: Changing views in perception. Trends in Cognitive Sciences, 3, 254-264

Lig, R., Wohlschlöger, A. M., Burazanis, S., Wöller, A., Nunnemann, S., \& Mühlau, M. (2008). Neural correlates of spontaneous percept switches in ambiguous stimuli: An event-related functional magnetic resonance imaging study. Cognitive Neuroscience, 28, 23252332.

Long, G. M., \& Toppino, T. C. (2004). Enduring interest in perceptual ambiguity: Alternating views of reversible figures. Psychological Bulletin, 130(5), 748-768.

Luo, J., \& Niki, K. (2003). Function of Hippocampus in "insight" of problem solving. Hippocampus, 13, 316-323.
Oh, S. (2009). The reversible limbs of a stick walker. Perception, 38 , $149-152$.

Pearson, J., \& Brascamp, J. (2008). Sensory memory for ambiguous vision. Trends in Cognitive Sciences, 12, 334-341.

Peterson, M. A., \& Hochberg, J. (1983). Opposed-set measurement procedure: A quantitative analysis of the role of local cues and intention in form perception. Journal of Experimental Psychology: Human Perception and Performance, 9, 183-193.

Pomerantz, J., \& Kubovy, M. (1986). Theoretical approaches to perceptual organization. In K. R. Boff, L. Kaufman, \& J. P. Thomas (Eds.), Handbook of perception and human performance, Vol. 2. Cognitive Processes and Human Performance (pp. 36-31-36-46). New York: Wiley.

Reisberg, D. (1983). General mental resources and perceptual judgments. Journal of Experimental Psychology: Human Perception and Performance, 9, 966-979.

Rock, I. (1975). An introduction to perception. New York: Macmillan.

Rock, I. (1983). The logic of perception. Cambridge: MIT Press.

Rock, I., Hall, S., \& Davis, J. (1994). Why do ambiguous figures reverse? Acta Psychologica, 87, 33-59.

Rock, I., \& Mitchener, K. (1992). Further evidence of failure of reversal of ambiguous figures by uninformed subjects. Perception, 21, 3945.

Sterzer, P., Frith, C., \& Petrovic, P. (2008). Believing is seeing: expectations alter visual awareness. Current Biology, 18, R697R698.

Sterzer, P., Kleinschmidt, A., \& Ress, G. (2009). The neural bases of multistable perception. Trends in Cognitive Sciences, 13, 310 318.

Vanrie, J., Dekeyser, M., \& Verfaillie, K. (2004). Bistability and biasing effects in the perception of ambiguous point-light walkers. Perception, 33, 547-560. 